\title{
A BAER-KAPLANSKY THEOREM FOR MODULES OVER PRINCIPAL IDEAL DOMAINS
}

\author{
SIMION BREAZ
}

\begin{abstract}
We will prove that if $G$ and $H$ are modules over a principal ideal domain $R$ such that the endomorphism rings $\operatorname{End}_{R}(R \oplus G)$ and $\operatorname{End}_{R}(R \oplus H)$ are isomorphic, then $G \cong H$. Conversely, if $R$ is a Dedekind domain such that two $R$-modules $G$ and $H$ are isomorphic whenever the rings $\operatorname{End}_{R}(R \oplus G)$ and $\operatorname{End}_{R}(R \oplus H)$ are isomorphic, then $R$ is a PID.
\end{abstract}

1. Introduction. The Baer-Kaplansky theorem states that two primary abelian groups with isomorphic endomorphism rings are necessarily isomorphic, [6, Theorem 108.1]. This statement was extended to various classes of modules (abelian groups), e.g., in $[8,14,16,20,21]$. However, straightforward examples show that in order to obtain such extensions we need to impose restrictions on these classes. For instance, the endomorphism rings of the Prüfer group $\mathbb{Z}\left(p^{\infty}\right)$ and of the group of $p$-adic integers $\widehat{\mathbb{Z}}_{p}$ are both isomorphic to the ring $J_{p}$ of $p$-adic integers. This fact suggests that we need to restrict to some good classes of modules in order to obtain a Baer-Kaplansky type theorem. Such a result (valid for torsion-free modules over valuation domains) was proved in [21]. It is well known that the Baer-Kaplansky theorem cannot be extended to torsion-free groups (of rank 1) since there are infinitely many pairwise non-isomorphic torsion-free groups of rank 1 whose endomorphism rings are isomorphic to $\mathbb{Z},[\mathbf{1}]$. However, similar results to the Baer-Kaplansky theorem hold for some special classes of torsion-free groups, see [2]. In the setting of modules over complete valuation domains, May proved a theorem, [15, Theorem 1], for reduced modules which are neither torsion nor torsion-free and have a nice subgroup $B$

2010 AMS Mathematics subject classification. Primary 13G05, 16D70, 16S50, $20 \mathrm{~K} 30$.

Keywords and phrases. Endomorphism ring, principal ideal domain, cancellation property.

Research supported by the CNCS-UEFISCDI grant PN-II-RU-TE-2011-3-0065.

Received by the editors on July 9, 2013, and in revised form on November 15, 2013. 
such that $M / B$ is totally projective: if $M$ is such a module and $N$ is an arbitrary module such that they have isomorphic endomorphism rings, then $M \cong N$.

The main aim of this note is to prove a Baer-Kaplansky theorem for arbitrarily modules over principal ideal domains (Theorem 2.1): if $R$ is a (commutative) principal ideal domain, then the correspondence (from the class of $R$-modules to the class of rings)

$$
\Phi: G \longmapsto \operatorname{End}_{R}(R \oplus G)
$$

reflects isomorphisms of endomorphism rings. Moreover, this property characterizes principal ideal domains in the class of Dedekind domains: if $R$ is a Dedekind domain such that the correspondence $\Phi$ reflects isomorphisms, then $R$ is a PID. The restriction to Dedekind domains is motivated by the fact that these domains have the cancellation property, i.e., the endofunctor $R \oplus-: \operatorname{Mod}-R \rightarrow \operatorname{Mod}-R$ on the category of all $R$-modules reflects isomorphisms:

Theorem 1.1. [12, Proposition 3.6]. Let $R$ be a Dedekind domain. If $M$ and $N$ are two $R$-modules such that $R \oplus M \cong R \oplus N$, then $M \cong N$.

We need this property in order to obtain that $\Phi$ reflects isomorphisms (cf., Remark 2.5). However, in order to obtain such a correspondence which reflects isomorphisms, the cancellation property is not enough, as is proved in Proposition 2.6 (in contrast with the similar problem for subgroup lattices, approached in [3, Lemma 2]).

2. A Baer-Kaplansky theorem. The main result proved in this note is the following.

Theorem 2.1. Let $R$ be a Dedekind domain. The following are equivalent:

(1) The ring $R$ is a principal ideal domain:

(2) If $G$ and $H$ are $R$-modules such that $G^{\prime}=R \oplus G$ and $H^{\prime}=$ $R \oplus H$ have isomorphic endomorphism rings, then $G$ and $H$ are isomorphic. 
Proof. $(1) \Rightarrow(2)$. Let $e$ and $f$ be the idempotents in $\operatorname{End}_{R}\left(G^{\prime}\right)$ which are induced by the direct decomposition $G^{\prime}=R \oplus G$. Using the version for principal ideal domains of [6, Theorem 106.1], we observe that there are isomorphisms

$$
e \operatorname{End}_{R}\left(G^{\prime}\right) f \cong \operatorname{Hom}_{R}(G, R)
$$

and

$$
f \operatorname{End}_{R}\left(G^{\prime}\right) e \cong \operatorname{Hom}_{R}(R, G) \cong G
$$

If $\varphi: \operatorname{End}_{R}\left(G^{\prime}\right) \rightarrow \operatorname{End}_{R}\left(H^{\prime}\right)$ is an isomorphism, then the idempotents $\bar{e}=\varphi(e)$ and $\bar{f}=\varphi(f)$ induce a direct decomposition $H^{\prime}=B \oplus K$, where $B=\bar{e}\left(H^{\prime}\right)$ and $K=\bar{f}\left(H^{\prime}\right)$. By [6, 106(d)], there is an isomorphism $\operatorname{End}_{R}(B) \cong R$. Moreover, as before, we have the isomorphisms (of $R$-modules)

$$
\operatorname{Hom}_{R}(K, B) \cong \bar{e} \operatorname{End}_{R}\left(H^{\prime}\right) \bar{f} \cong \operatorname{Hom}_{R}(G, R),
$$

and

$$
\operatorname{Hom}_{R}(B, K) \cong \bar{f} \operatorname{End}_{R}\left(H^{\prime}\right) \bar{e} \cong \operatorname{Hom}_{R}(R, G) \cong G
$$

We claim that $B \cong R$. Using this and Theorem 1.1 we obtain $H \cong K$, and we have

$$
H \cong \operatorname{Hom}_{R}(R, K) \cong \operatorname{Hom}_{R}(B, K) \cong \operatorname{Hom}_{R}(R, G) \cong G .
$$

In order to prove our claim, suppose that $B \nsubseteq R$. Let $\alpha: B \rightarrow R$ be an $R$-homomorphism. Since $R$ is a PID it follows that $\operatorname{Im}(\alpha) \cong R$; hence, $\operatorname{Ker}(\alpha) \neq 0$. Moreover, $\operatorname{Im}(\alpha)$ is a projective module; hence, we have a direct decomposition $B \cong \operatorname{Ker}(\alpha) \oplus \operatorname{Im}(\alpha)$. But $\operatorname{End}(B) \cong R$ has no non-trivial idempotents; hence, $B$ is indecomposable. It follows that $\operatorname{Im}(\alpha)=0$; hence, $\operatorname{Hom}_{R}(B, R)=0$.

If we consider the direct decomposition $H^{\prime}=R \oplus H$ and the canonical projection $\pi_{R}: H^{\prime} \rightarrow R$, it follows that $B$ is contained in $H$, the kernel of $\pi_{R}$. From $H^{\prime}=B \oplus K$, we obtain $H=(H \cap K) \oplus B$. Using the equalities

$$
K \oplus B=R \oplus H=R \oplus(H \cap K) \oplus B,
$$


we deduce that $K \cong R \oplus(H \cap K)$ (as complements for the direct summand $B$ ); hence, $K$ has a direct summand isomorphic to $R$. Therefore, $\operatorname{Hom}_{R}(G, R) \cong \operatorname{Hom}_{R}(K, B)$ has a direct summand isomorphic to $B$. Since $R$ is commutative, $\operatorname{Hom}_{R}(G, R)$ is an $R$-module which can be embedded as a submodule in the direct product $R^{G}$ of copies of $R$ (here we view $R^{G}$ as the set of all maps $G \rightarrow R$, endowed with pointwise addition and scalar multiplication; see [5, Exercise 43.1]). Therefore, we can embed $B$ in $R^{G}$. Since $B \neq 0$, it follows that we can find a projection $\pi: R^{G} \rightarrow R$ such that $\pi(B) \neq 0$. This $\operatorname{implies} \operatorname{Hom}_{R}(B, R) \neq 0$, a contradiction, and it follows that $B \cong R$.

$(2) \Rightarrow(1)$. Let $I$ be a non-zero ideal in $R$. Since $R$ is Noetherian and integrally closed, we can apply [7, Theorem I.3.7] to conclude that $\operatorname{End}_{R}(I) \cong R$. Moreover, since $I$ is invertible, we can use the Steinitz isomorphism formula, [7, page 165]. Therefore, for every positive integer $n$, we have an isomorphism $\left(\oplus_{k=1}^{n-1} R\right) \oplus I^{n} \cong \oplus_{k=1}^{n} I$; hence, there are ring isomorphisms

$$
\begin{aligned}
\operatorname{End}_{R}\left(\left(\oplus_{k=1}^{n-1} R\right) \oplus I^{n}\right) & \cong \operatorname{End}_{R}\left(\oplus_{k=1}^{n} I\right) \cong M_{n}(R) \\
& \cong \operatorname{End}_{R}\left(\oplus_{k=1}^{n} R\right) .
\end{aligned}
$$

If $n \geq 2$, we obtain, from (2) that $\left(\oplus_{k=1}^{n-2} R\right) \oplus I^{n} \cong \oplus_{k=1}^{n-1} R$. Using again the cancellation property of $R$, Theorem 1.1, we conclude that $I^{n}$ is principal for all $n \geq 2$. If $C(R)$ is the ideal class group associated to $R$ and $[I]$ is the class of $I$ in this group, it follows that $[I]^{n}=1$ for all $n \geq 2$; hence, $[I]=1$. Then $I$ is principal and the proof is complete.

Remark 2.2. From the above proof, it follows that if $R$ is a principal ideal domain, then every ring isomorphism $\varphi: \operatorname{End}_{R}(R \oplus G) \rightarrow$ $\operatorname{End}_{R}(R \oplus H)$ induces a direct decomposition $R \oplus H=B \oplus K$ with $B=\varphi(e)(R \oplus H) \cong R$ and $(1-\varphi(e))(R \oplus H)=K \cong G$, where $e$ is the idempotent such that $e(R \oplus G)=R$ and $(1-e)(R \oplus G)=G$. Since $B \cong R$, it is not hard to see, using the same technique as in the proof for the bounded case of [6, Theorem 108.1], that $\varphi$ is induced by an isomorphism $\psi: R \oplus G \rightarrow R \oplus H$. Therefore, the above theorem can be viewed as an improvement of [19, Theorem 2.1] for the case of principal ideal domains.

Remark 2.3. A class $\mathcal{C}$ of modules is called Baer-Kaplansly if any two of its modules are isomorphic whenever their endomorphism rings are 
isomorphic as rings, [9, p. 1489]. Therefore, Theorem 2.1 says that the class of modules over a Dedekind domain $R$ which have a direct summand isomorphic to $R$ is a Baer-Kaplansky class if and only if $R$ is a principal ideal domain. Similar results for other kinds of rings were obtained in [8, Theorem 8] for a similar class, respectively, in [9, Theorem 4] for a particular class of modules over FGC-rings.

As a consequence of Theorem 2.1, we obtain that locally free modules over principal ideal domains are determined by their endomorphism rings. This is also a consequence of $[21$, Theorem A].

Corollary 2.4. If two locally free modules over a principal ideal domain have isomorphic endomorphism rings, then they are isomorphic.

Remark 2.5. In the proof of Theorem 2.1 we used the cancellation property of the regular module $R$. If $R$ does not have this property (e.g., there are Dedekind-like domains without the cancellation property, [10]) then there are two $R$-modules $G \nsubseteq H$ such that $R \oplus G \cong R \oplus H$; hence, $\operatorname{End}_{R}(R \oplus G) \cong \operatorname{End}_{R}(R \oplus H)$. If we write these endomorphism rings as matrix rings,

$\operatorname{End}_{R}(R \oplus G)=\left(\begin{array}{cc}\operatorname{End}_{R}(R) & \operatorname{Hom}_{R}(G, R) \\ \operatorname{Hom}_{R}(R, G) & \operatorname{End}_{R}(G)\end{array}\right) \cong\left(\begin{array}{cc}R & \operatorname{Hom}_{R}(G, R) \\ G & \operatorname{End}_{R}(G)\end{array}\right)$, respectively,

$$
\operatorname{End}_{R}(R \oplus H) \cong\left(\begin{array}{cc}
R & \operatorname{Hom}_{R}(H, R) \\
H & \operatorname{End}_{R}(H)
\end{array}\right)
$$

we observe that the $(2,1)$-blocks in these representations are isomorphic to $G$, respectively, to $H$. These two blocks are not isomorphic even when the corresponding matrix rings are isomorphic. It is obvious that, in this case, Theorem 2.1 is not valid.

We will prove that we cannot replace in the implication $(1) \Rightarrow(2)$ of Theorem 2.1 the direct summand $R$ by an arbitrary module which has the cancellation property. The following proposition shows that the property of the regular module $R$ stated in Theorem 2.1 is stronger than the usual cancellation property (see $[\mathbf{1 8}$, Theorem B]). 
Proposition 2.6. The following are equivalent for an indecomposable torsion-free abelian group $F \neq 0$ of finite rank:

(1) If $G$ and $H$ are abelian groups such that $\operatorname{End}(F \oplus G) \cong$ $\operatorname{End}(F \oplus H)$, then $G \cong H$;

(2) $F \cong \mathbb{Z}$.

Proof. $(1) \Rightarrow(2)$. If $F$ is not isomorphic to $\mathbb{Z}$, then $F \cong \mathbb{Q}$ or $F$ is a reduced abelian group which has no free direct summands.

For the case $F \cong \mathbb{Q}$, we can choose $G$ and $H$ as two non-isomorphic subgroups of $\mathbb{Q}$ such that $\operatorname{End}(G)=\operatorname{End}(H)=\mathbb{Z}$. It is not hard to see that both endomorphism rings $\operatorname{End}(F \oplus G)$ and $\operatorname{End}(F \oplus H)$ are isomorphic to the matrix $\operatorname{ring}\left(\begin{array}{ll}\mathbb{Q} & 0 \\ \mathbb{Q} & \mathbb{Z}\end{array}\right)$, so $F$ does not verify condition (1).

If $F$ is a reduced abelian group which has no free direct summands, then we can construct, using [18], two (reduced) finite rank torsion-free groups $G$ and $H$ of the same rank such that

$$
\operatorname{Hom}(F, G)=\operatorname{Hom}(F, H)=\operatorname{Hom}(G, F)=\operatorname{Hom}(H, F)=0
$$

and

$$
\operatorname{End}(G)=\operatorname{End}(H)=\mathbb{Z} .
$$

In this case, both endomorphism $\operatorname{rings} \operatorname{End}(F \oplus G)$ and $\operatorname{End}(F \oplus H)$ are isomorphic to the ring $\operatorname{End}(F) \times \mathbb{Z}$, so $F$ does not verify condition (1).

$(2) \Rightarrow(1)$. This is a consequence of Theorem 2.1 .

Remark 2.7. There are also versions for the Baer-Kaplansky theorem proved for automorphism groups, Jacobson radicals or for ring antiisomorphisms, $[\mathbf{4}, \mathbf{1 1}, \mathbf{1 3}, \mathbf{1 7}]$. It would be nice to know if Theorem 2.1 is still true if we consider only automorphism groups or Jacobson radicals.

Acknowledgments. I would like to thank professor Luigi Salce for his help in proving the implication $(2) \Rightarrow(1)$ in Theorem 2.1.

\section{REFERENCES}

1. D.M. Arnold, Finite rank torsion free Abelian groups and rings, Lect. Notes Math. 931, Springer-Verlag, 1982. 
2. E. Blagoveshchenskaya, G. Ivanov and P. Schultz, The Baer-Kaplansky theorem for almost completely decomposable groups, Contemp. Math. 273 (2001), 85-93.

3. S. Breaz and G. Călugăreanu, Every Abelian group is determined by a subgroup lattice, Stud. Sci. Math. Hung. 45 (2008), 135-137.

4. A.L.S. Corner, B. Goldsmith and S.L. Wallutis, Anti-isomorphisms and the failure of duality, in Models, modules and abelian groups, Walter de Gruyter, 2008.

5. L. Fuchs, Infinite Abelian groups I, Academic Press, New York, 1970.

6. - Infinite Abelian groups II, Academic Press, New York, 1973.

7. L. Fuchs and L. Salce, Modules over non-Noetherian domains, Math. Surv. Mono. 84, American Mathematical Society, Providence, RI, 2001.

8. G. Ivanov, Generalizing the Baer-Kaplansky theorem, J. Pure Appl. Alg. 133 (1998), 107-115.

9. G. Ivanov and P. Vámos, A characterization of FGC rings, Rocky Mountain J. Math. 32 (2002), 1485-1492.

10. W. Hassler and R. Wiegand, Direct sum cancellation for modules over onedimensional rings, J. Alg. 283 (2005), 93-124.

11. J. Hausen, C. Praeger and P. Schultz, Most abelian p-groups are determined by the Jacobson radical of their endomorphism rings, Math Z. 216 (1994), 431-436.

12. T.Y. Lam, A crash course on stable range, cancellation, substitution and exchange, J. Alg. Appl. 3 (2004), 301-343.

13. H. Leptin, Abelsche p-Gruppen und ihre Automorphismengruppen, Math. Z. 73 (1960), 235-253.

14. W. Liebert, Endomorphism rings of free modules over principal ideal domains, Duke Math. J. 41 (1974), 323-328.

15. W. May, Isomorphism of endomorphism algebras over complete discrete valuation rings, Math. Z. 204 (1990), 485-499.

16. F. Richman and E.A. Walker, Primary abelian groups as modules over their endomorphism rings, Math. Z. 89 (1965), 77-81.

17. P. Schultz, A. Sebeldin and A.L. Sylla, Determination of torsion Abelian groups by their automorphism groups, Bull. Aust. Math. Soc. 67 (2003), 511-519.

18. J. Stelzer, A cancellation criterion for finite-rank torsion-free Abelian groups, Proc. Amer. Math. Soc. 94 (1985), 363-368.

19. K. Wolfson, Isomorphisms between endomorphism rings of modules, Proc. Amer. Math. Soc. 123 (1995), 1971-1973.

20. Anti-isomorphisms of endomorphism rings of locally free modules, Math.Z. 202 (1989), 151-159.

21. K.G. Wolfson, Isomorphisms of the endomorphism rings of torsion-free modules, Proc. Amer. Math. Soc. 14 (1963), 589-594.

Babeş-Bolyai University, Faculty of Mathematics and Computer Science, Str. Mihail Kogălniceanu 1, 400084 Cluj-Napoca, Romania

Email address: bodo@math.ubbcluj.ro 